Received Date : 27-Oct-2015

Revised Date : 07-Apr-2016

Accepted Date : 17-Apr-2016

Article type : Original Article

\title{
The results of local excision with or without postoperative adjuvant chemoradiotherapy for early rectal cancer among patients choosing to avoid radical surgery
}

Authorship

Initials \& Surname Qualifications Main Appointment Institution

Svetlana BalyasnikovaMD, PhD Radiology Clinical/ Research Fellow The Royal Marsden

Hospital, NHS Foundation trust,

Imperial College London

James Read MSc, FRCS SpR in General Surgery $\quad$ Croydon University Hospital, NHS Foundation trust

Diana Tait MD, FRCR Consultant in Clinical Oncology The Royal Marsden

Hospital, NHS Foundation trust

Andrew Wotherspoon MB BCh, FRCPath Consultant Histopathologist The Royal Marsden Hospital, NHS Foundation trust

Ian Swift MD, FRCS Consultant Colorectal Surgeon Croydon University

Hospital, NHS Foundation trust

David Cunningham MD, FRCP Professor of GI Medicine and Lymphoma The Royal Marsden Hospital, NHS Foundation trust

Paris Tekkis MD, FRCS Professor of Colorectal Surgery The Royal Marsden

Hospital, NHS Foundation trust and Imperial College London

This article has been accepted for publication and undergone full peer review but has not been through the copyediting, typesetting, pagination and proofreading process, which may lead to differences between this version and the Version of Record. Please cite this article as doi: 10.1111/codi.13477

This article is protected by copyright. All rights reserved. 


\title{
Gina Brown MD, FRCR Professor of GI Cancer Imaging
}

Hospital, NHS Foundation trust and Imperial College London

\section{Corresponding Author: \\ Name: Prof Gina Brown \\ Email address: Gina.Brown@rmh.nhs.uk}

Twitter: @prof_gina_brown

Conflict of interest: None to declare

\begin{abstract}
Aim: The study aimed to establish the oncological outcome of patients who opted for close surveillance with or without adjuvant chemoradiotherapy (CRT) rather than radical surgery after local excision (LE) of early rectal cancer (ERC)

Method: The Royal Marsden Hospital Rectal Cancer database was used to identify rectal cancer patients treated by primary LE from 2006-2015. All patients were entered in an intensive surveillance programme.

Results: Twenty eight of 34 analysed patients had a high or very high risk of residual disease predicted by adverse histopathological features for which the recommendation had been radical surgery. Eighteen (52\%) of the 34 had received radiotherapy following LE. Three-year disease free survival for the 34 patients was $85 \%$ (95\% CI 78.8-91.2\%) and overall survival was $100 \%$. Twenty two of 24 patients with a low tumour which would have required total rectal excision have so far avoided radical surgery and remain disease free at a median follow up 3.2 years.

Conclusion: The findings suggest that with modern MRI and clinical surveillance radical surgery can be avoided in patients following initial LE of a histopathologically defined high risk ERC. These findings are comparable with those obtained after major radical resection and warrant further prospective investigation as a treatment arm in larger prospective trials.
\end{abstract}

This article is protected by copyright. All rights reserved. 
Key words: early rectal cancer, adjuvant chemotherapy after local excision

What does this paper add to the literature?

Under current guidelines patients with a locally excised "high risk" early rectal cancer are advised to have a completion major resection to avoid disease recurrence. For patients who decline, the results of the current study suggest that MRI and colonoscopic surveillance with selective chemoradiotherapy may allow avoidance of major rectal surgery with low rates of disease relapse at three years.

\section{Introduction}

The UK national bowel cancer screening programme is increasing the proportion of colorectal cancers diagnosed at an early stage. Logan et al published the results of the first one million screened patients and reported $42 \%$ of screen detected colorectal cancers to be stage I (1). Early rectal cancer (ERC) can be defined as a Stage I, Dukes A or TNM T1 or T2 N0 cancer. Local excision (LE) not compromising the oncological outcome could potentially be offered to the majority of such patients. The technical options for LE vary depending on the facilities and expertise available in the unit and include endoscopic mucosal or submucosal resection and transanal surgical techniques including transanal endocopscopic microsurgery (TEM) and transanal resection (TAR). About half of sessile malignant polyps resected have adverse features on histopathological assessment such as poor differentiation, mucinous-type histology, lymphovascular invasion or direct invasion deep into the submucosal layer (2). Such features are associated with a $20 \%$ rate of lymph node metastasis. In this circumstance the current standard of practice is to offer a radical resection to avoid leaving residual locoregional disease (3). An anterior resection with total mesorectal excision (TME) after local excision may be difficult owing to inflammation and fibrosis especially if it is delayed,. Furthermore, patients may not accept such major surgery with its mortality and morbidity particularly if there is a high probability that there will be no residual tumour in the resection specimen.

Investigating ERC to define the role of both local excision and adjuvant therapy was the most important research priority agreed by the recent Delphi survey of over 900 colorectal surgeons of the Association of Coloproctology of Great Britain and Ireland (ACPGBI) (4). Previously published data do not support the use of adjuvant chemoradiotherapy (CRT) after local excision of high risk T1 or T2 cancers as an alternative to completion major resection. Paty et al report that adjuvant CRT after local excision only delays the time to local recurrence from 1.1 years to 2.1 years which was $7 \%$ for 
$\mathrm{T} 1$ and $24 \%$ for T2 in their unit (5). The prospective phase II study by Greenberg et al reported ten year rates of disease-free survival of $75 \%$ and $64 \%$ for patients with locally excised T1 and T2 lesions (6). This study did not, however, use magnetic resonance imaging (MRI) or ultrasound to stage the tumour before treatment but instead relied on computerized tomography (CT), carcinooembrionic antigen (CEA) estimation and clinical assessment (6) and the protocol of follow up after local excision was not stipulated.

In recent years there have been improvements in the assessment of early rectal cancer and surveillance by high-resolution MRI and also in the technique of local excision. In our practice patients with early rectal cancer treated by LE undergo high-resolution MRI to identify any extramural macroscopic disease, such as malignant lymph nodes or extramural vascular invasion,. In accordance with national guidelines the policy in our hospital was to offer completion radical surgery if adverse histology features were reported after local excision (3). If radical surgery was declined we gave patients the alternative option of adjuvant chemo-radiotherapy and intensive follow up. Here we report on our experience, our surveillance protocol and the oncological outcomes of such patients with adverse histology who opted for follow up with or without adjuvant therapy rather than completion radical surgery.

\section{Method}

\section{Patients}

The study was registered as a service evaluation protocol and approved by the hospital research data management and statistical unit. The Royal Marsden Hospital Rectal Cancer database was used to identify rectal cancer patients treated by local excision from 2006-2015. All patients referred to the Royal Marsden MDT who underwent LE or TEM for a rectal lesion proven to be adenocarcinoma were included. Patients were excluded if they had had preoperative CRT before local excision. Thirty-six consecutive patients were found applying the above-mentioned criteria, but two patients who had a piecemeal resection of a dysplastic adenoma with no confirmation oc malignancy on histopathology were excluded from the final analysis. there were therefore 34 (22 male) patients of mean age 63 (39-83) years with an early rectal carcinoma (ERC) who were eligible for analysis (Figure 1).

\section{Local Excision}

Local excision was performed by transanal endoscopic microsurgery (TEM) or transanal resection (TAR). The indications included diagnostic and therapeutic. It was carried out according to the preoperative features of the tumour as follows:

This article is protected by copyright. All rights reserved. 
i) for tumours considered to be malignant with suspected invasion of less than the full thickness of the muscularis propria (MP), a full thickness local excision by transanal microrsurgery (TEM) or transanal resection $[\mathrm{TAR}])$ was carried out.

ii) for tumours not considered to be invasive on clinical assessment, a local excision with submucosal or partial or full thickness resection of the MP was performed with diagnostic intent, which then turned out to be the final treatment after histopathological examination of the surgical specimen.

The initial decision to perform local excision was based predominantly on clinical assessment by digital rectal examination and rectoscopy and took into account the further factors of biopsy findings, size of lesion, patient preference, comorbidity and the feasibility of local excision. The recommendation to offer radical surgery was based on the histopathological assessment of the local excision surgical specimen taking into account known prognostic factors for recurrence after local excision, in accordance with the ACPGBI guidelines (3).

\section{Adjuvant therapy}

When completion surgery was declined by the patient but high risk features were considered present, adjuvant CRT was offered and 18/34 (53\%) of patients chose to have post local excision external beam radiotherapy (EBRT). Treatment was delivered by a conformal technique and the dose fractionation depended on the histopathological features and the estimated local recurrence risk. For patients with a clear margin on the local excision specimen, where microscopic involvement of mesorectal lymph nodes was perceived as the main risk, 45Gy in 25 fractions were given to the pelvis to include the mesorectum with concomitant Capecitabine $825 \mathrm{mg} / \mathrm{m} 2$ twice daily. Where there was an involved or close margin in the local excision specimen, the tumour bed was boosted with further of EBRT delivering 9Gy in 5 fractions to five and 5.4Gy to 3 fractions to two patients.

\section{Surveillance}

Patients who received adjuvant CRT were followed up by the Clinical Oncology team at three monthly intervals for the first two years and thereafter at six monthly intervals to five years. An examination was carried out at every clinic visit with the results of MRI and CEA available at each visit. An annual CT scan was performed according to usual protocol for patients with colorectal cancer. Patients having local excision without adjuvant chemoradiation were followed at similar intervals by the surgical team.

This article is protected by copyright. All rights reserved. 


\section{Magnetic resonance imaging}

MR was performed on 1.5T MR systems (Siemens) using a body-matrix phased array surface coil centred over the pelvis. After localizer sequences, high-resolution T2 weighted TSE scans were acquired in coronal and sagittal planes, followed by high-resolution (HR) oblique-axial scans (perpendicular to the long axis of the rectum with $160 \mathrm{~mm}$ FOV, $3 \mathrm{~mm}$ slice thickness, no inter slice gap and matrix 256x256).

We used the routine reporting proforma of the radiological department at The Royal Marsden Hospital. The site of local excision was determined, by interpreting low signal intensity as the scar, regardless of whether it was confined to the rectal wall or extended into the mesorectum. Irregular intermediate signal intensity at the site was interpreted as showing the presence of residual or recurrent tumour. The craniocaudal length and thickness of the excised area was measured, assuming that any progressive increase in size or thickness of the scar or treated tumour site or new intermediate signal intensity within the mesorectum was suggestive of recurrence. If recurrence or persistence of tumour was suspected in the lumen or the mesorectum on either MRI or clinical assessment, the patient was discussed in the MDT.

\section{Statistical analysis}

Data were expressed as median and range or as a proportion of the denominator of 34 with the 95\% confidence interval (CI). Overall survival (OS) was calculated measured from the date of local excision to the most recent follow up date or death from any cause. The disease free survival (DFS) was calculated from the date of surgery to the point of the appearance of metastatic or local disease,or cancer related death. Survival curves for OS and DFS were obtained using the Kaplan-Meier method. Patients without an event were censored at the last follow up. A p-value of $<0.05$ was taken as being statistically significant. Statistical analysis was performed using SPSS version 19.0.

\section{Results}

Patients

Local excision wa undertaken in 34 patients. In 19 the procedure was diagnostic and in the remaining 15 patients it was therapeutic. Twelve (58\%) of the 19 and $13(82 \%)$ of the 15 patients had had a preoperative MRI. Six in the diagnostic and three in the therapeutic group had TEM. Eighteen patients were referred from the local network of hospitals where MRI for ERC was agreed policy and of these 17 (94\%) had had a preoperative MRI (Table 1).

This article is protected by copyright. All rights reserved. 


\section{Histopathology}

The final histology of the 34 surgical specimens was as follows: pT1 Sm1-2; 16 (47\%) , pT1 Sm3; 10 (29\%), pT2; 7 (21\%), pT3b; 1(3\%) (Table 2). The resection margin was less than $1 \mathrm{~mm}$ in 18 (53\%) of the 34 specimens and $1-2 \mathrm{~mm}$ in five (15\%) with missing data in 7 (21\%). Intramural lymphovascular invasion (LVI) was identified in 13 (38\%). This increased with the depth of invasion being present in $28.5 \%$ of $\mathrm{T} 1 \mathrm{Sm} 2$ tumours , $40 \%$ of $\mathrm{T} 1 \mathrm{sm} 3$ tumours and $57 \%$ of $\mathrm{T} 2$ tumours. Information on tumour budding status was missing in nine patients.

Poorly differentiated tumours were found in $9 \%$ of cases and a further $23 \%$ showed mucin histology. Therefore of the 34 patients undergoing local excision, 28 (82\%) were categorized into group of high or very high risk for local recurrence according to ACPGBI recommendations (3).

Treatment

In $25(73 \%)$ patients the lower border of the tumour was less than $6 \mathrm{~cm}$ from the anal verge. In 24 (71\%) the MDT recommended abdominoperineal excision of the rectum (APER). This was based on assessment of the potential surgical plane of dissection required to achieve clearance of more than $1 \mathrm{~mm}$. Overall the MDT advised radical surgery or adjuvant chemoradiotherapy in 28 (82\%) patients, but 18 opted to be treated by adjuvant CRT and surveillance and 10 declined CRT and chose clinical and MRI surveillance. In the patients opting for adjuvant CRT and close surveillance, the median interval from the local excision to starting CRT was 62.5 (30-136) days.

Patients were analysed in three groups according to the management strategy after local excision These included i) 18 patients at high-risk of local recurrence who received adjuvant CRT (Group 1), ii) 10 patients who opted for surveillance with no adjuvant CRT (Group 2) and iii) 6 patients at low risk for local recurrence (Group 3). The median follow up for all patients was 49 (19-121) months. The overall survival (OS) at 4.0 years was $100 \%$. At a median follow-up interval of 49 (14-121) months (Table 3) none of the patients in the management Groups 1 and 3 developed local or distant recurrence.

Meanwhile two patients who declined further treatment group (the second group in the survival curve analysis) were identified as having residual extramural disease and macroscopic residual tumour at the site of local excision on the first surveillance MRI and colonoscopy and underwent radical surgery. In both cases radical surgery, consisted of APE, was performed within three months after primary LE. The final histopathological examination of the surgical specimen confirmed pT3bNOR0 
and T2NOR0 adenocarcinoma. None of these patients developed distant metastases. A further two patients of the second group developed local recurrence at 6 and 7 months after LE surgery, and one (10\%) developed distant lung metastases at 11 months (Figure 1).

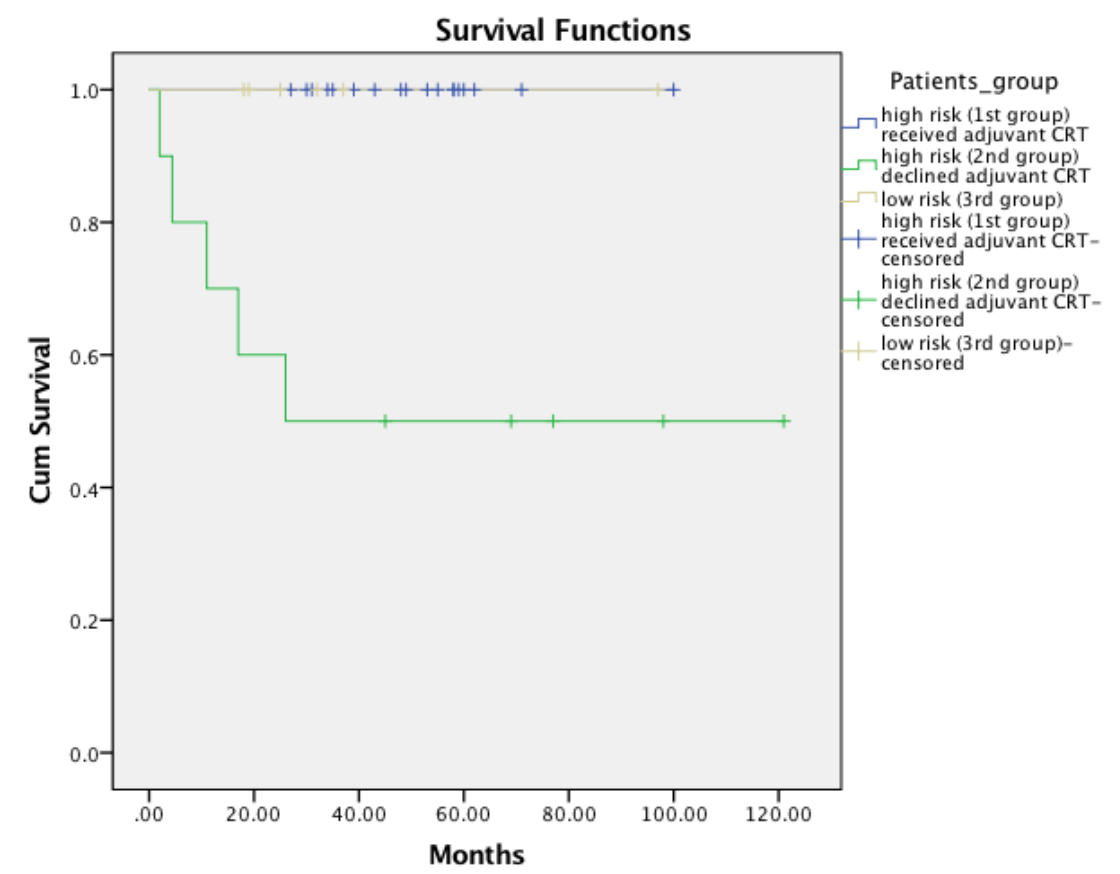

Fig. 1. Disease free survival after local excision

One patient experienced a local recurrence at seven months after local excision. Histopathological examination had shown a pT1sm2 tumour containing mucin. The patient had had no adjuvant CRT and an extramural mucinous recurrence was diagnosed on a routine follow-up MRI scan (Figure 2). The patient underwent APE for ypT2N0 tumour, and remains under surveillance without pelvic recurrence or distant metastasis.

The other case of local recurrence occurred in a patient treated by a LE for yT1sm3R1 moderately differentiated adenocarcinoma. Following the actual excision a defect in the rectal wall, which appeared to be abutting the mesorectal fascia was seen. The patient declined any further treatment and underwent close surveillance. A six months MRI scan showed a new area of nodularity with intermediate signal intensity at the site of the surgical scar. The patient subsequently received CRT which was commenced 247 days after LE. An MRI scan immediately following this treatment 
showed a good response, but a subsequent MRI within 3 months of the end of adjuvant CRT showed tumour regrowth which was confirmed by rectal biopsy to be carcinoma. The patient eventually underwent an APE and has no evidence of recurrent or metastatic disease at 82 months from the initial LE.
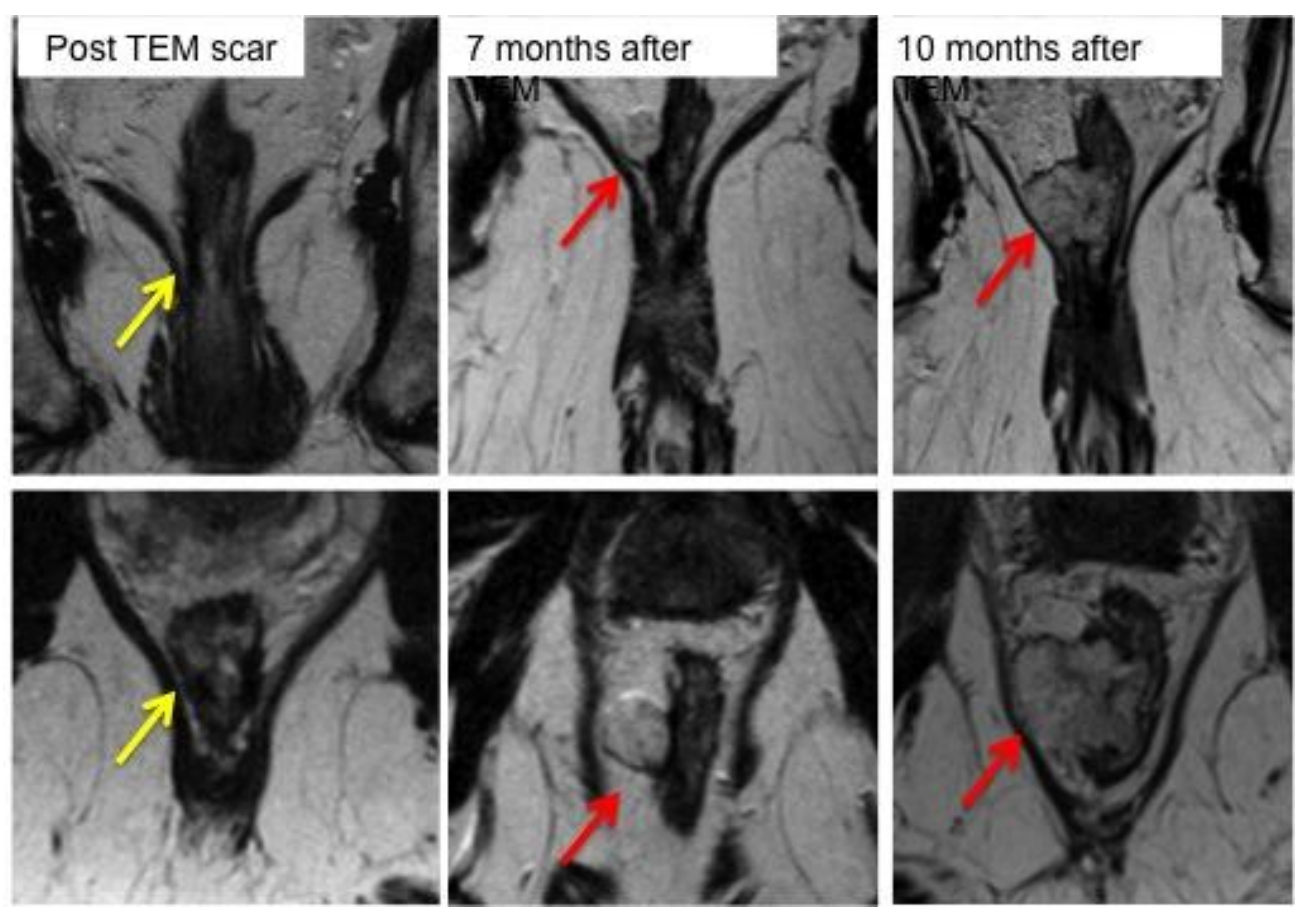

Fig. 2. A post-TEM MRI shows a well-defined scar (yellow arrow) at the site of excision of a low rectal pT1sm2 tumour containing mucin. At seven months after surgery an MRI scan demonstrated an area of extramural hyperintense signal at the site of the TEM scar, highly suspicious of local recurrence. Three months later disease progression was noted and extramural mucinous mass had now infiltrated the rectal wall at the site of the scar. A biopsy confirmed recurrence. MRI successfully had identified relapse before clinical evidence of recurrence by 7 months).

One patient from the $2^{\text {nd }}$ group developed a pulmonary metastasis within 11 months after local excision of a poorly differentiated pT1sm 3 adenocarcinoma. The metastasis was resected with no further sites of relapse in the 36 months following local excision.

This article is protected by copyright. All rights reserved. 


\section{Discussion}

The present study demonstrated that patients undergoing local excision of an early rectal cancer with histopathological features indicating a high risk of recurrence who opted for adjuvant CRT and close surveillance rather than major surgery, had no recurrence either local or distant and a three year disease free survival of $100 \%$. In contrast patients with a high-risk locally excised tumour, who chose close surveillance but no further treatment, experienced a local recurrence rate of $40 \%$. Overall 22 (92\%) of the 24 patients included in the study successfully avoided a recommended APE for at least 3 years.

We acknowledge that the study is not a prospective trial but rather an analysis of a prospective patient database. We do not have data of the outcome of patients with tumours of a similar stage who opt for completion major surgery as in our practice, most patients opt for surveillance. We also do not have experience of significant numbers of patients undergoing surveillance without adjuvant therapy. Nevertheless, the safety of completion major resection after local excision of early rectal cancer with a high histopathological risk of recurrence has been reported by others to be very low. Thus Hahnloser et al reported a local recurrence rate of $3 \%$ after completion major resection following the local excision of high risk ERC which was no different from the $5 \%$ rate of local recurrence after primary radical surgery (7). Radical surgery in early rectal cancer after a previous local excision performed by whatever technique can be technically difficult owing to the presence of fibrosis and inflammation. Adjuvant chemoradiotherapy in patients at high risk of local recurrence after LE is not included in the published treatment guidelines and appears to have been considered an alternative option rather than standard treatment as there are insufficient data to support this approach. The most recently published systematic review on ERC included ten studies that evaluated local excision combined with adjuvant CRT. This showed that at a range of 30.5-115.2 months median overall survival (OS) was 75 (66-80.6) \% and DFS was 74 (64-85.2) \% (8), although some of the older studies included in the review had not used modern MRI for the initial assessment and the subsequent follow up.

The present study has shown that when a patient having local excision of a high risk ERC declines recommended major surgery, close surveillance is effective. Thus the single patient who developed metastatic disease was identified in our surveillance programme and underwent early metastectomy and the four patients with local recurrence underwent abdominoperineal excision with curative intent. In all of these patient local recurrence was identified on high resolution MRI and confirmed by biopsy. MRI is superior to endoscopy for assessing the local excision scar during follow up as it can visualise the extraluminal component and the whole mesorectum and pelvic side wall can be monitored, . This may account for the improved safety and survival seen in the present series.

This article is protected by copyright. All rights reserved. 
Recurrence has been reported up to seven years after local excision and adjuvant therapy, but the vast majority of relapses occur in the first three years $(5,6)$. Contemporary trials evaluating local excision also reported the oncological endpoints at a three years. The findings of this study therefore raise the possibility of discussing surveillance rather than radical surgery as an initial option in patients with a locally excised ERC even one with high risk histopathological features.

Larger prospective studies with longer follow up are needed, but if the results of the present study are confirmed they would be a significant advance in the treatment of ERC. Institutions intending to study the question of management after LE for ERC should be confident of the quality of their tumour staging and surveillance programmes. For a rectal adenoma removed endoscopically and found to contain unexpected malignancy, the results of the present study agree with the literature that further therapy, whether CRT or surgery, can be avoided in a proportion of patients. It also suggests, however, that an ERC treated by local excision which contains adverse histopathological features should be considered for adjuvant CRT rather than having major surgery. Whatever option is chosen, close surveillance should be performed including high resolution pelvic MRI to exclude any residual or recurrent intra and extramural disease.

It is important to recognise that a comparable oncological outcome after subsequent major surgery or surveillance with or without CRT, must include the consideration of other factors including mortality, morbidity, function and quality of life. Selective adjuvant chemoradiotherapy based on carefully documented histopathology together with a policy of careful MRI-based surveillance, in patients having local excision for an early rectal cancer appears to be feasible and warrants evaluation by future research.

\section{Acknowledgements}

Karen Thomas, Lead statistican, The Royal Marsden Hospital, Sutton

This study was funded and supported by the Biomedical Research Centre

This article is protected by copyright. All rights reserved. 


\section{References}

1. Logan RF, Patnick J, Nickerson C, Coleman L, Rutter MD, von Wagner C, et al. Outcomes of the Bowel Cancer Screening Programme (BCSP) in England after the first 1 million tests. Gut. 2012;61(10):1439-46.

2. Seitz U, Bohnacker S, Seewald S, Thonke F, Brand B, Braiutigam T, et al. Is endoscopic polypectomy an adequate therapy for malignant colorectal adenomas? Presentation of 114 patients and review of the literature. Diseases of the colon and rectum. 2004;47(11):1789-96; discussion 967.

3. Williams JG, Pullan RD, Hill J, Horgan PG, Salmo E, Buchanan GN, et al. Management of the malignant colorectal polyp: ACPGBI position statement. Colorectal Dis. 2013;15 Suppl 2:1-38.

4. Tiernan J, Cook A, Geh I, George B, Magill L, Northover J, et al. Use of a modified Delphi approach to develop research priorities for the association of coloproctology of Great Britain and Ireland. Colorectal disease : the official journal of the Association of Coloproctology of Great Britain and Ireland. 2014;16(12):965-70.

5. Paty PB, Nash GM, Baron P, Zakowski M, Minsky BD, Blumberg D, et al. Long-term results of local excision for rectal cancer. Ann Surg. 2002;236(4):522-29; discussion 9-30.

6. Greenberg JA, Shibata D, Herndon JE, 2nd, Steele GD, Jr., Mayer R, Bleday R. Local excision of distal rectal cancer: an update of cancer and leukemia group B 8984. Dis Colon Rectum.

2008;51(8):1185-91; discussion 91-4.

7. Hahnloser D, Wolff BG, Larson DW, Ping J, Nivatvongs S. Immediate radical resection after local excision of rectal cancer: an oncologic compromise? Dis Colon Rectum. 2005;48(3):429-37.

8. Ung L, Chua TC, Engel AF. A systematic review of local excision combined with chemoradiotherapy for early rectal cancer. Colorectal Dis. 2014;16(7):502-15.

This article is protected by copyright. All rights reserved. 
Table 1. The number of patients who underwent diagnostic and therapeutic local excision

\begin{tabular}{|c|c|c|c|c|c|c|c|c|c|}
\hline \multirow[b]{2}{*}{$\begin{array}{l}\text { Preoperative } \\
\text { biopsy }\end{array}$} & \multirow{2}{*}{$\begin{array}{c}\text { Preoperative } \\
\text { MRI } \\
(\mathrm{Y} / \mathrm{N})\end{array}$} & \multirow{2}{*}{$\begin{array}{l}\text { Mucosal } \\
\text { resection }\end{array}$} & \multicolumn{2}{|c|}{$\begin{array}{l}\text { Full thickness } \\
\text { resection of } \\
\text { the rectal wall }\end{array}$} & \multicolumn{5}{|c|}{ pT stage } \\
\hline & & & TAE & TEM & pT1sm1 & pT1sm2 & pT1sm3 & pT2 & pT3b \\
\hline Benign & $12 / 7$ & 7 & 6 & 6 & 1 & 8 & 6 & 3 & 1 \\
\hline Malignant & $13 / 2$ & 5 & 7 & 3 & 1 & 6 & 4 & 4 & 0 \\
\hline Total & $25 / 9$ & 12 & 13 & 9 & 2 & 14 & 10 & 7 & 1 \\
\hline
\end{tabular}

$\mathrm{MRI}=$ magnetic resonance imaging, $\mathrm{LE}=$ local excision, $\mathrm{TAR}=$ transanal rexection, $\mathrm{TEM}=$ transanal endoscopic microsurgery,

Table 2. The results of histopathological examination of the excised local excision specimen

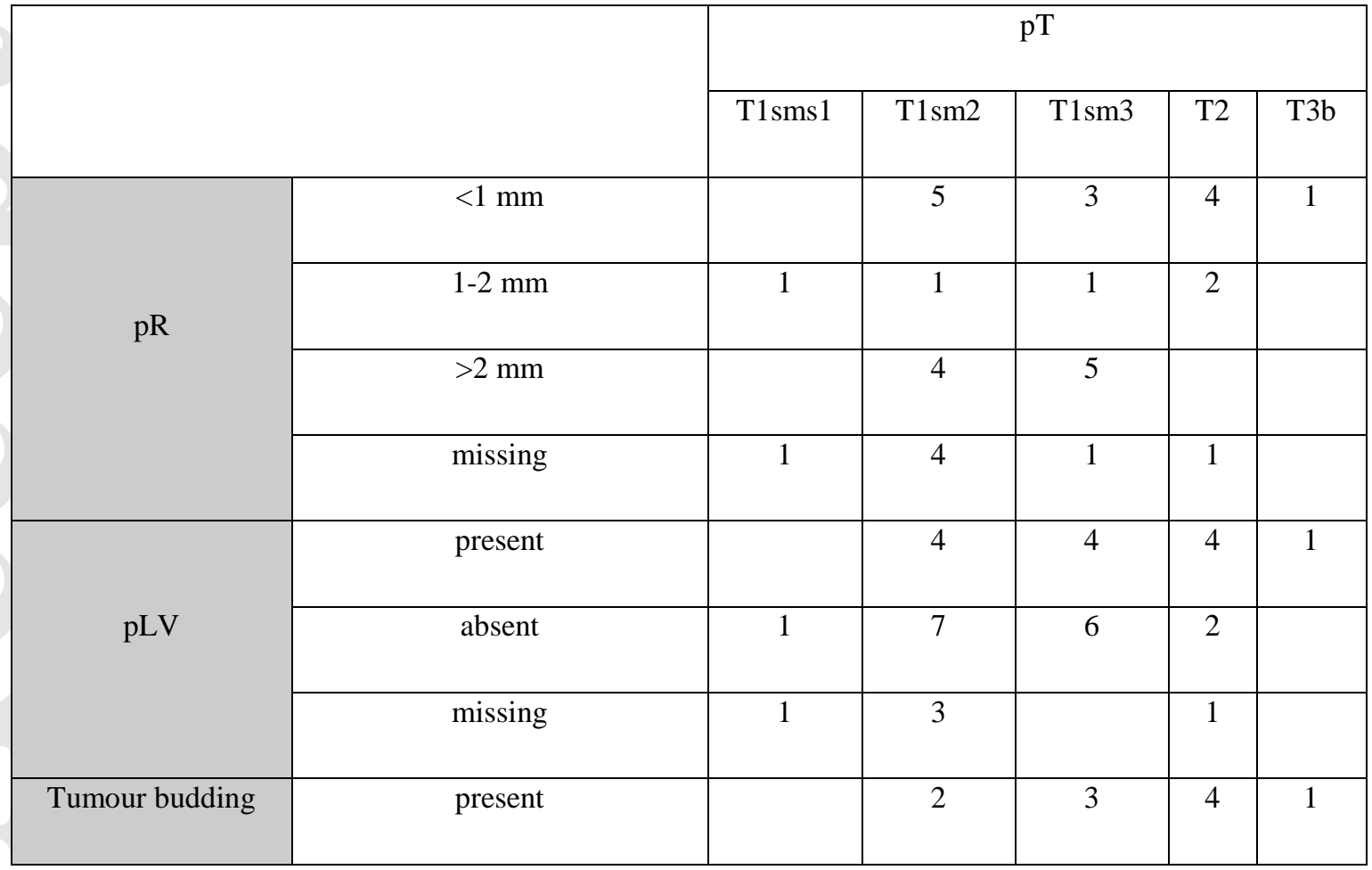

This article is protected by copyright. All rights reserved. 


\begin{tabular}{|c|c|c|c|c|c|c|}
\hline & absent & & 8 & 5 & 2 & \\
\hline & missing & 2 & 4 & 2 & 1 & \\
\hline \multirow{4}{*}{$\mathrm{pG}$} & well/moderately & 1 & 7 & 5 & 4 & \\
\hline & poorly & & & 2 & & 1 \\
\hline & mucinous & 1 & 3 & 2 & 2 & \\
\hline & missing & & 4 & 1 & 1 & \\
\hline \multirow{4}{*}{$\begin{array}{c}\text { Risk for local } \\
\text { recurrence }\end{array}$} & Low risk $(<5 \%)$ & 1 & 0 & 0 & 0 & 0 \\
\hline & Medium risk (5-10\%) & 1 & 4 & 0 & 0 & 0 \\
\hline & High risk $(8-15 \%)$ & 0 & 1 & 0 & 0 & 0 \\
\hline & Very-high risk $(>20 \%)$ & 0 & 9 & 10 & 7 & 1 \\
\hline \multirow{2}{*}{$\begin{array}{c}\text { Distance from the } \\
\text { anal verge }\end{array}$} & $>6 \mathrm{~cm}$ & 1 & 4 & 2 & 2 & \\
\hline & $<6 \mathrm{~cm}$ & 1 & 10 & 8 & 5 & 1 \\
\hline \multicolumn{2}{|c|}{ Adjuvant CRT } & 0 & 5 & 6 & 7 & 1 \\
\hline \multicolumn{2}{|c|}{ Local recurrence } & 0 & 1 & 1 & 0 & 0 \\
\hline \multicolumn{2}{|c|}{ Distant metastases } & 0 & 0 & 1 & 0 & 0 \\
\hline \multicolumn{2}{|c|}{ Total } & 2 & 14 & 10 & 7 & 1 \\
\hline
\end{tabular}

$\mathrm{pR}$ - resection margin

pLV-lymphovascular invasion

pG- tumour differentiation grading

This article is protected by copyright. All rights reserved. 
Table 3. Duration of follow up

\begin{tabular}{|l|c|c|c|c|c|c|c|c|c|}
\hline Months & $\mathbf{0}$ & $\mathbf{6}$ & $\mathbf{1 2}$ & $\mathbf{1 8}$ & $\mathbf{2 4}$ & $\mathbf{3 0}$ & $\mathbf{3 6}$ & $\mathbf{4 2}$ & $\mathbf{4 8}$ \\
\hline Total number of patients & 34 & 34 & 34 & 33 & 31 & 30 & 27 & 21 & 19 \\
\hline Number receiving CRT & 18 & 18 & 18 & 18 & 18 & 17 & 14 & 12 & 11 \\
\hline
\end{tabular}

This article is protected by copyright. All rights reserved. 


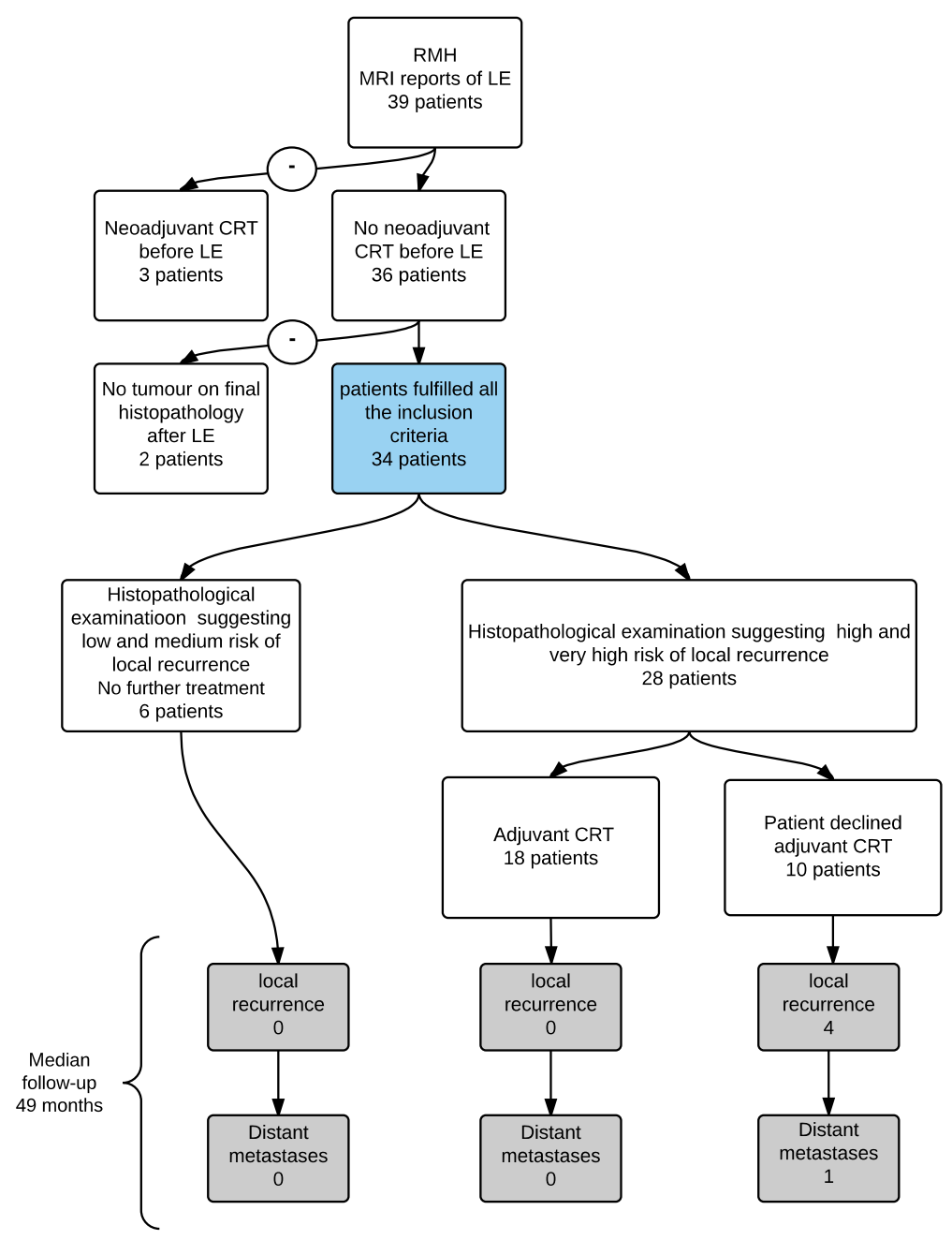

Figure 1: Flow chart of the study

$\mathrm{RMH}=$ Royal Marsden Hospital, MRI = magnetic resonance imaging, $\mathrm{LE}=$ local excision

$\mathrm{CRT}=$ chemoradiotherapy

This article is protected by copyright. All rights reserved. 
High-resolution MRI protocol for the follow-up after local excision

Scar at site of primary excision

Identification of scar

Height $(\mathrm{mm})$ of scar from anal verge

Longitudinal length ( $\mathrm{mm}$ )

Thickness (mm)

Is there evidence of intermediate signal intensity in the scar or within or outside the rectal wall

\section{Nodal Spread:}

Mesorectum imaged at high resolution up to the level of S1

Benign appearing nodes (yes/no)?

Are morphologically suspicious lymph nodes identified within the mesorectum?

Has there been a change from previous MRI?

\section{Malignant pelvic sidewall nodes}

Other pelvic viscera:

Do the pelvic viscera appear morphologically normal?

\section{Pelvic peritoneum}

Is there MR evidence of pelvic peritoneal disease?

Bony pelvis:

Suspicious bony lesions? (yes/no)

Opinion

MR evidence or no evidence of locoregional recurrence

This article is protected by copyright. All rights reserved. 